\title{
DIPHTHERIA CARRIERS AND THEIR TREATMENT WITH MERCUROCHROME
}

\author{
George A. Gray and Bertha I. MEYer \\ From the U. S. Naval Hospital, Mare Island, Cal.
}

Diphtheria being a preventable disease, the sudden outbreak of three cases in as many days in the Lock Ward of the U. S. Naval Hospital, Mare Island, Calif., caused the following steps to be taken to check its spread. All patients, personnel and civilian employes in the hospital compound were immediately examined and Schick tests, nose and throat cultures were made. Of 544 Schick tests, 104, or 19.1\%, were found to be positive. These susceptible men were immediately given 1000 units of diphtheria antitoxin and a course of three injections of toxinantitoxin was commenced. All new patients brought to the hospital were first taken to the laboratory where cultures were made and where they received the Schick test before they were admitted to their wards. Regarding the cultures made of those in the hospital compound, special care was taken to enter thoroughly the nasal passages and to extend the swab well back to the nasopharynx. In the throat an attempt was made to have the swab enter the tonsillar. crypts and the pharynx. The cultures were then grown on Loeffler's medium, and the smears stained by Neisser's method and examined carefully.

A rather high percentage of carriers of diphtheria bacilli were found, 162 of 680 persons examined being positive. The majority of positive reactions were secured from the nasal passages, and we believe were in no small part due to the thoroughness used in making the cultures, by having the swabs come in contact with all parts of the mucous membranes. Our percentage of 23.8 is considerably in excess of the $2.76 \%$ found in 3,215 persons examined at Camp Sherman, Ohio, in 1918. ${ }^{1}$ It conforms, however, fairly closely with the figures of Labit of the French army, who obtained $28.9 \%$, and at times $50 \%$, of carriers in the environment of diphtheria at a military hospital. ${ }^{2}$ We consider that there is an environment of diphtheria at this station as the disease is practically endemic in the nearby towns.

This large number of carriers was so distributed throughout the various hospital wards and personnel that to have isolated them all

Received for publication Jan. 4, 1921.

1 McCord, Friedlander and Walker: Jour. Am. Med. Assn., 1818, 71, p. 275.

${ }^{2}$ Arch. de méd. et de pharm. mil., 1917, 67, p. 779. 
would have seriously interfered with the functioning of the hospital. As every one connected with the hospital was immunized against diphtheria, it was decided not to isolate the carriers but rather to attempt to make them carrier free just as soon as possible, by conducting them through a thorough and systematic course of treatment. In looking over the various methods of treating carriers the results were rather discouraging, and there seemed to be no particular method of choice other than to use some germicide and to apply it thoroughly and systematically to the mucous membranes of the nose and throat. We therefore decided that the first rule governing our treatment of the carriers would be to enforce a consistent, thorough and systematic application of whatever germicide or agent we chose to use, to the walls of the nose and throat. In this way we hoped to have the germicide reach, as far as was in our power, the hiding place of the organisms. The problem of treating the carriers was placed in the hands of the laboratory staff, who had the facilities for making the necessary cultures at intervals, to check up on the treatment. All ambulatory carriers were therefore to report to the laboratory for treatment at 8:00 a. m. and 5:00 p. m. daily, while bed carriers were to be treated in their wards by a representative of the laboratory. The minimum standard set, before a patient was considered as being carrier free, was three consecutive negative cultures taken at 48-hour intervals, the third culture being secured at least 24 hours after the last local treatment. The cultures were made in the morning before treatment.

In searching for a germicide, we had to consider the enemy we were engaged against, knowing full well his predilection for hiding places in crypts of the tonsil, between the folds of the mucous membranes, in erosions, ulcers and also behind any shelter he can find in the nasal and pharyngeal passages. As a matter of fact, we considered that $\dot{w}$ e were facing a situation somewhat similar to the one that the genitourinologist is confronted with, in trying to have his antiseptic solutions reach the gonococcus, as it establishes itself beneath the cells and in the glands, lining the mucous membrane of the urethra. The report by Young, White and Swartz, ${ }^{3}$ therefore, on the uses of the new germicide mercurochrom-220 (a bisodium salt of dibrom-oxymercury-fluorescein), lauding the marked penetrating and germicidal properties of that drug, and demonstrating that it can be utilized in relatively concentrated solutions on the mucous membranes of the bladder and urethra

3 Jour. Am. Med. Assn., 1919, 78, p. 1483. 
without causing irritation, gave us the suggestion to use this same drug germicide in an attempt to reach the diphtheria bacilli in their various hiding places in the nose and throat. We, therefore, used aqueous solutions of mercurochrome in $1 / 2,1$ and $2 \%$ strengths.

Of our total number of carriers we were able to treat, consecutively, thoroughly and systematically, only 90 . The remaining were treated more or less itinerently, and have not been included in our figures regarding the efficacy of mercurochrome in the treatment of diphtheria carriers. One or two of the medical officers, some of the nurses and several of the patients obtained the idea that our procedure was to be more or less of an experiment, and we encountered some lack of cooperation in the use of this germicide. As a result, therefore, for those who objected to receiving the mercurochrome, we used any one of a number of substances, such as diphtheria antitoxin, argyrol, normal salt solution, etc.; and as they appeared only at irregular intervals, we obtained results no different from those usually obtained in treating carriers by the old and more or less established procedures, as noted later. Then, too, 16 of our carriers were discharged to duty from the hospital. Five others absconded, and 8 more were found to be in the civilian Chinese help, and it was next to impossible to give them any kind of systematic treatment. They were treated whenever we could reach them. This leaves 51 of our carriers who were not treated systematically with mercurochrome. Of this number, we are still treating 35 with various antiseptics as mentioned and 16 have gone to duty as being carrier free. This treatment has been going on for about 35 days so that by the time the condition of our 35 remaining carriers clears up, they will have a number of sick days far in excess of the average number for the carriers treated with mercurochrome.

Eighty-eight of our 90 carriers treated with mercurochrome were made carrier free with an average of only 19.1 treatments, or applications of the germicide, with an average of but 12.7 sick days. The 2 extra sick days (19.1 treatments approximating 10 days) resulted from one day in the hospital without treatment before his last culture had been made, and another day passing before we were able to obtain the result of this final culture. The remaining 2 of the 90 carriers resisted all our efforts as they continued to harbor the bacilli in tonsillar crypts and in the scars of a peritonsillar abscess. One of these two was even treated by having him lie on his side, flooding the tonsillar area with a $2 \%$ solution of mercurochrome, and retaining it there for 10 
or 15 minutes, but without avail. We finally had to resort to tonsillectomy in this case. This patient's nose, which was also positive (a clinical case), cleared up relatively early under the use of mercurochrome, but the throat, although we obtained an occasional negative culture, consistently remained positive.

In not over 10 persons treated with mercurochrome solution was there any complaint as to the germicide being irritating. Several of these persons who were sensitive to the drug were medical officers and nurses. They described it as a feeling of congestion in the nose. One nurse complained of a marked dryness of the mucous membranes of the nasal and pharyngeal passages, but we discovered that she had the same sensation from the application of antitoxin and other antiseptics. In the latter case, we resorted to normal salt solution, and she was speedily relieved of symptoms as well as of diphtheria bacilli.

The $1 \%$ solution of mercurochrome was used as a routine application by means of a medicine dropper, spray or swab. In the more resistant cases we used the $2 \%$ strength, and in cases showing any evidences of congestion or irritation, we used the $0.5 \%$ strength. When persons complained of the application of the drug by means of the nasal swab, we used the medicine dropper and had them hold their heads back for a minute or two after the application, until the drug passed back into the nasopharynx. We relied on the medicine dropper in the nasal tract almost entirely and on the swab for the tonsils. In patients with nasal obstructions we utilized the nasal spray as a means to have the germicide reach the organisms.

\section{CONCLUSIONS}

Our high percentage of diphtheria carriers conforms to those of Labit of the French army for men in a diphtheria environment.

In addition to systematizing the treatment of diphtheria carriers, we have found that the germicide of choice is a solution of mercurochrome220 in $0.5,1$ or $2 \%$ strength. By using this drug we were able to obtain in our series of carriers of diphtheria bacilli 88 carrier-free persons, following an average of 19.1 applications of this solution, with an average of only 12.7 sick days, as compared to an average of 23 days, the best results we have found recorded for a large number of carriers. 